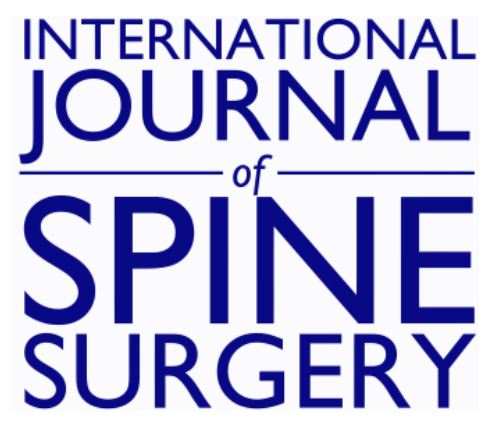

\title{
Understanding the Aorta-Spine Relation in Idiopathic Scoliosis: Value of Noncontrast CT-Derived Curved Coronal Reformats and 3D Volume Images
}

Khaled M. Zaghloul, Ahmed M. Tawfik, Basma Gadelhak, Hoda M. Sobh, Nihal M. Batouty and Donia M. Sobh

Int J Spine Surg 2021, 15 (4) 818-825

doi: https://doi.org/10.14444/8105

http://ijssurgery.com/content/15/4/818

This information is current as of April 26, 2023.

Email Alerts Receive free email-alerts when new articles cite this article. Sign up at: http://ijssurgery.com/alerts 


\title{
Understanding the Aorta-Spine Relation in Idiopathic Scoliosis: Value of Noncontrast CT-Derived Curved Coronal Reformats and 3D Volume Images
}

\author{
KHALED M. ZAGHLOUL, MD, ${ }^{1}$ AHMED M. TAWFIK, MD, ${ }^{2,3}$ BASMA GADELHAK, MD ${ }^{2}$ HODA M. \\ SOBH, MD,${ }^{4}$ NIHAL M. BATOUTY, MD ${ }^{2}$ DONIA M. SOBH, $\mathrm{MD}^{2}$ \\ ${ }^{1}$ Department of Orthopaedic Surgery, Mansoura University, Faculty of Medicine, Mansoura, Egypt, ${ }^{2}$ Department of Diagnostic and Interventional Radiology, \\ Mansoura University, Faculty of Medicine, Mansoura, Egypt, ${ }^{3}$ Department of Radiology, Andalusia Hospital AlShalalat, Andalusia Group for Medical Services, \\ Alexandria, Egypt, ${ }^{4}$ Department of Cardiology, Mansoura University, Faculty of Medicine, Mansoura, Egypt
}

\begin{abstract}
Background: Investigating axial position and longitudinal bending of the aorta in relation to spine curvature in adolescent idiopathic scoliosis patients could help surgeons in planning of spine surgeries.

Methods: Noncontrast computed tomography (CT) scans of 27 consecutive patients with adolescent idiopathic scoliosis (19 right and 8 left curves) and 16 control subjects were retrospectively reviewed. Using semiautomated software, centerline was drawn along the descending aorta, and curved reformat was generated. Aorta tortuosity index (TI) was calculated as (centerline length/straight line distance) $-1 \times 100$. The spine centerline was drawn from T1 to L5, and curve index (CI) was similarly calculated. The aorta centerline angle was measured. Apical vertebral-rotation angle and multilevel aorto-vertebral angles were measured on axial CT. Three-dimensional volume-rendered images of the aorta were generated using a manual region grow function.

Results: Mean ( \pm standard deviation) Cobb's angle was $63.8 \pm 34.6^{\circ}$. The spine CI of patients $(9.7 \pm 7.11)$ was significantly higher than controls $(0.28 \pm 0.22), P=.00001$. Aorta TI in scoliosis was significantly higher than controls $(6.4 \pm 7.2$ versus $0.6 \pm 0.5, P=.0001)$. The aorta centerline angle was steeper in scoliosis than controls $\left(140 \pm 26.8^{\circ}\right.$ versus $\left.170 \pm 3.6^{\circ}\right)$. Correlations were excellent between the aorta TI and each of Cobb's angle, spine CI, and vertebral rotation angle $(r=0.851$ to 0.867 , all $P<.001)$. Aorto-vertebral angles were significantly different between right scoliosis and left scoliosis patients and control groups at T6, T7, T8, L2, and L3 levels.

Conclusions: Aortic curvature increases in proportion to the degree of scoliosis. The aorta follows the concavity of scoliosis in right and left curves. In the axial CT plane, the aorta in both right and left scoliosis is maximally rotated away from its normal position at T7 and is closest to its normal position at T11 to T12.
\end{abstract}

Clinical Relevance: Quantitative evaluation of aortic curvature combined with preoperative reconstructed CT images could be beneficial for surgeons in planning of spine surgeries.

Other \& Special Categories

Keywords: scoliosis, aorta, adolescent idiopathic scoliosis, computed tomography, spine

\section{INTRODUCTION}

The anatomic relation between the aorta and the spine in patients with adolescent idiopathic scoliosis has been the subject of several studies for more than 2 decades. ${ }^{1-10}$ The reason for this enthusiasm is the conceivable risk for aortic injury during surgical correction, either when placing anterior instrumentation $^{3,4,11}$ or posterior pedicle screws. ${ }^{6,7,12}$ Axial plane computed tomography (CT) or magnetic resonance images were used to assess the position of the aorta in relation to the spine using several reference aorto-vertebral angles and aorta-spine distance measurements, with or without comparison to normal subjects, to delineate the safety of surgical interventions. ${ }^{1-12}$ Few studies have also analyzed the same relations after surgical correction to determine the proximity of the aorta to the placed instrumentation. ${ }^{4,13,14}$

Change in the position of the aorta relative to the spine in scoliosis is not only a function of vertebral rotation, because the aorta does not remain fixed, but is also related, at least in part, to the accompanying change in the course of the aorta itself, as it assumes a curved course rather than its normal straight course.

In contrast to the well-investigated aorta-spine relationship in the axial plane, ${ }^{1-14}$ bending of the 
aorta in the longitudinal plane in scoliosis patients did not receive enough attention in the literature. The pattern of aortic bending and how proportional it is to the degree of scoliosis have not been reported yet.

Preoperative CT imaging assessment of aortovertebral relation is recommended before scoliosis corrective surgeries to avoid early and late aortic injuries. ${ }^{15}$ Scoliosis patients are usually scanned without intravenous contrast, which is the reason why multiplanar and $3 \mathrm{D}$ reconstructions of the aorta are not obtained. However, in our experience, it is feasible to obtain adequate quality $3 \mathrm{D}$ volumerendered images displaying the aorta in addition to the spine from noncontrast CT images using commercially available software and in convenient time. These sets of images could be useful as a roadmap for the surgeon especially in anterior instrumentation, as they give an overview of the whole course of the aorta and its relative position to the whole spine simultaneously.

The aim of this work was to reconstruct 3D volume-rendered images and curved multiplanar reformats of the aorta from noncontrast CT scans to investigate the longitudinal bending of the descending aorta in relation to the curvature of the spine in adolescent idiopathic scoliosis patients compared with normal control subjects. Qualitative and quantitative analyses of the aortic longitudinal curve were performed.

\section{METHODS}

\section{Study Population}

This retrospective study was approved by our Institutional Review Board (decision/protocol number MS.20.03.1082), and patient consent was waived. The study population included 27 consecutive patients with idiopathic adolescent scoliosis, 19 with right curve and 8 with left curve. Their preoperative multidetector noncontrast CT scans of the whole spine were retrospectively evaluated. Seventeen consecutive CT scans of the thorax and abdomen were collected from the database of our hospital (with or without contrast) in patients with similar age range and normal spine, to represent the control group.

\section{Image Analysis}

Images were transferred to an offline workstation (IntelliSpace Portal V5, Philips Healthcare). Commercially available semiautomated software was used to draw a centerline along the aorta; the start point was just after the origin of the left subclavian artery, and the end point was at the aortic bifurcation. From this centerline, curved reformat of the aorta was automatically generated and manually adjusted when necessary. The centerline length and the straight-line length of the aorta between the start and end points were documented in millimeters. Similarly, curved reformat for the spine was obtained, and the centerline and straight-line for the spine from $\mathrm{T} 1$ to $\mathrm{L} 4$ were measured in millimeters (Figure 1). On 3D volume-rendered images of the spine, the manual region grow function was applied to display the aorta; the time taken to obtain the 3D reconstructions was recorded.

The following parameters were obtained and are shown in Figures 1 and 2:

1. The tortuosity index (TI) of the aorta was calculated as (centerline length/straight line length) $-1 \times 100$ (Figure 1a). ${ }^{16}$

2. The curve index (CI) of the spine was calculated as (centerline length/straight line length) $-1 \times 100$ (Figure 1b).

3. The centerline angles for the aorta and spine were measured on the curved reformat using semiautomated software for aneurysm stent insertion function (Figure 1c).

4. Vertebral rotation angle (Figure 2a): on axial images at the apex of scoliosis, between 2 lines intersecting at the dorsal center of the vertebral foramen, 1 line joins this point to the anterior midline of the chest wall, and the other line runs through the midline of the vertebral body.

5. Aorto-vertebral angle at each level from T4 to $\mathrm{L}^{4}$ (Figure 2b): from axial images, the angle subtended by the vertical orthogonal line passing through the center of the vertebral body and a line passing through the center of the aorta. Thus, the angle was zero when the aorta was strictly anterior to the midvertebral body, $90^{\circ}$ when the aorta was directly lateral to the left, and $-90^{\circ}$ when the aorta was directly to the right of spine.

6. The Cobb's angle was documented from patients' medical files.

\section{Statistical Methods}

All statistics were performed using statistical software (IBM SPSS version 22.0). Continuous data 

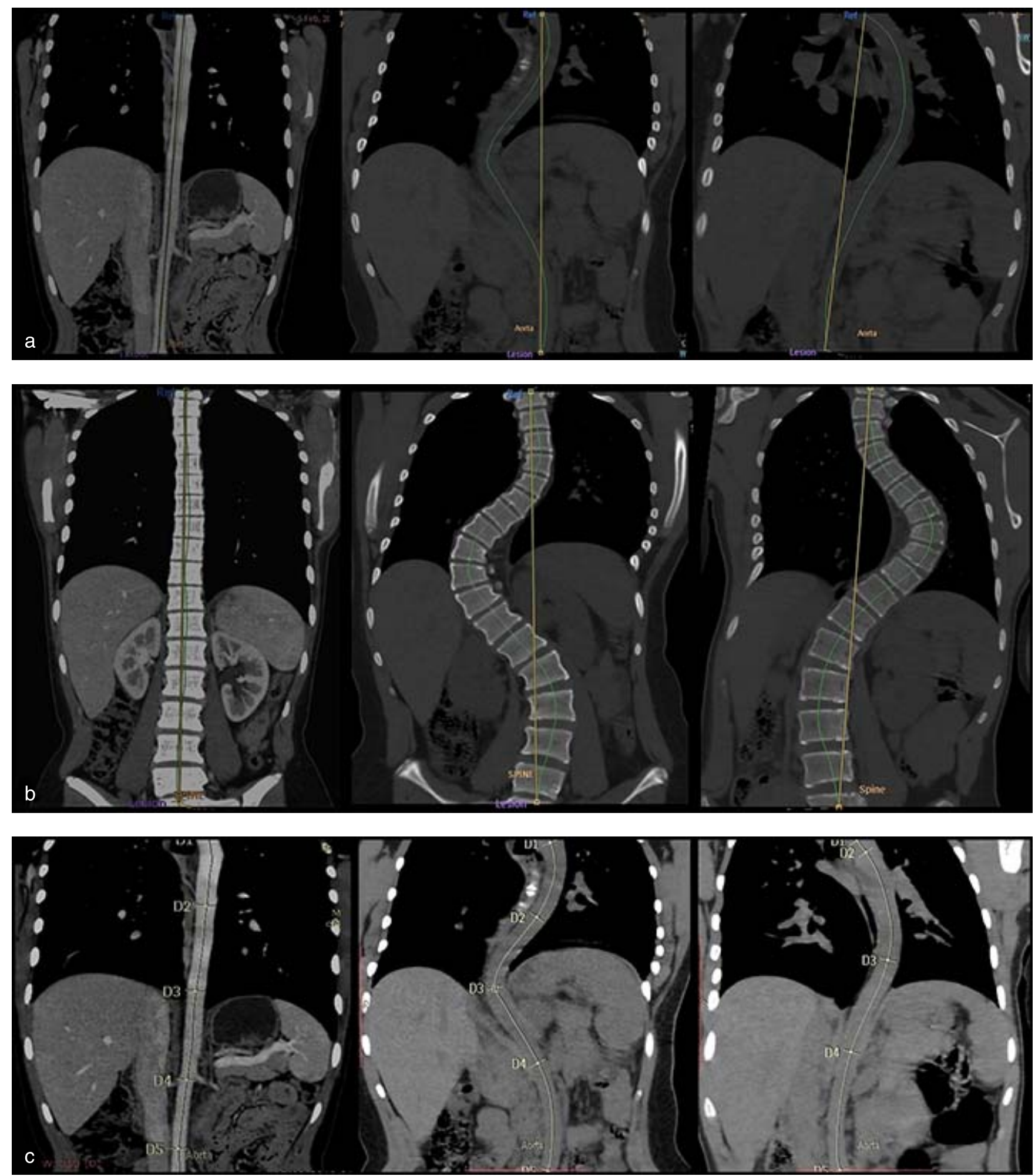

Figure 1. Examples of the measured parameters on curved reformatted computed tomography images in a control subject (left), scoliosis patient with right curve (middle), and scoliosis patient with left curve (right). (a) Tortuosity index of the aorta. (b) Curve index of the spine. (c) Centerline angle of the aorta.

were presented as mean \pm standard deviation $(\mathrm{SD}) /$ median and range. A Kolmogorov-Smirnov test was performed to assess data normality. Student's $t$ test or a Mann-Whitney test (parametric or nonpara- metric data, respectively) was used to compare the mean/median of aorta TI, aorta centerline angle, and spine $\mathrm{CI}$ between adolescent idiopathic scoliosis patients and controls. Correlations were performed 

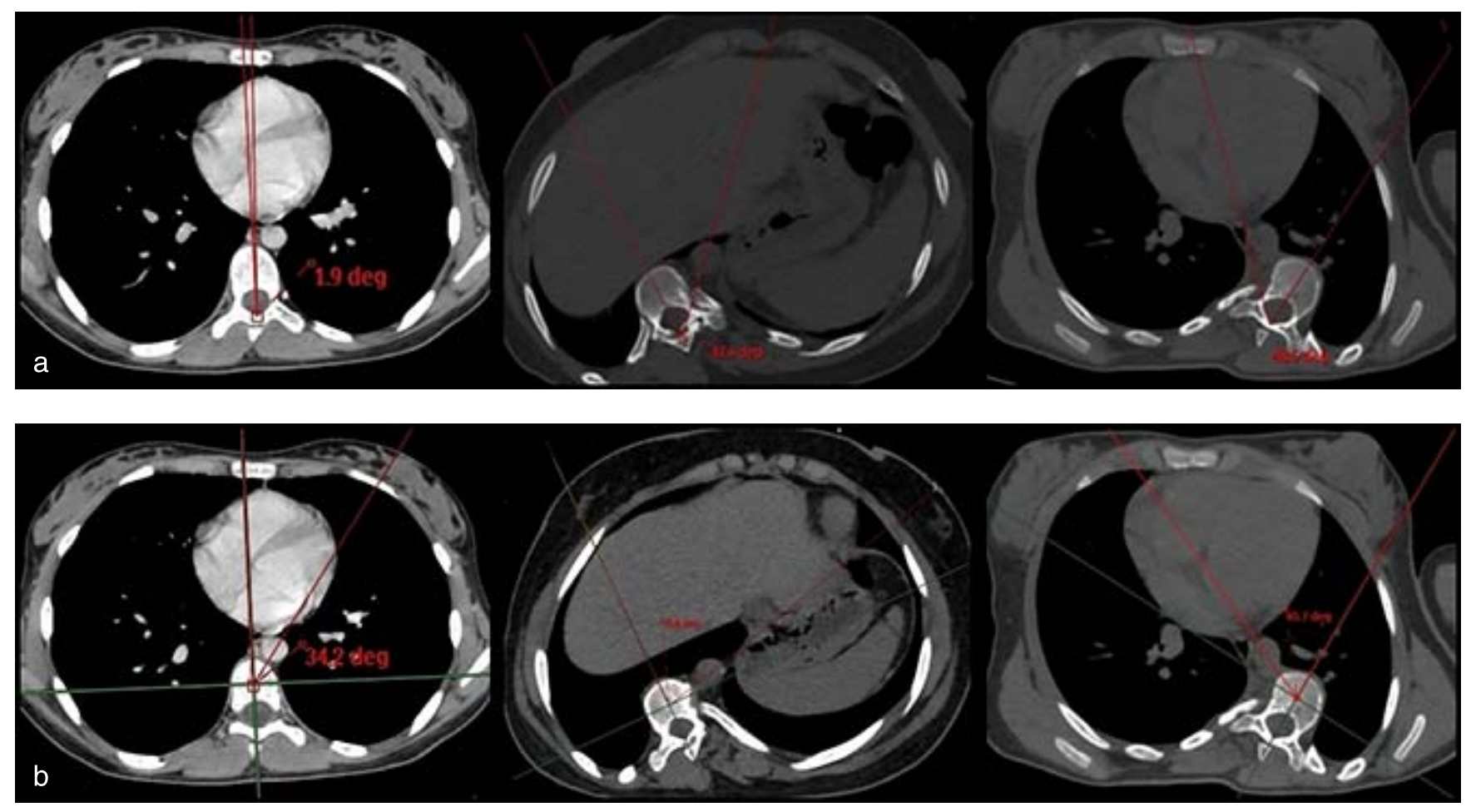

Figure 2. Examples of the measured angles on axial computed tomography images in a control subject (right), scoliosis patient with right curve (middle), and scoliosis patient with left curve (left). (a) Vertebral rotation angle. (b) Aorto-vertebral angle.

using a Pearson correlation test. The means of multilevel aorto-vertebral angles were compared between right curve scoliosis patients, left curve scoliosis patients, and the control group using a oneway analysis of variance test. $P$ values $<.05$ were considered statistically significant.

\section{RESULTS}

The patient group consisted of 18 females and 9 males (median age 15 years, range 11 to 22 years), and the control group consisted of 12 females and 6 males (median age 16 years, range 12 to 23 years), with no significant difference in age between the 2 groups $(P=.06)$.

In the patient group, the scoliotic curve was right in 19 patients and left in 8 patients. The mean $( \pm$ SD) Cobb's angle was $63.8 \pm 34.6^{\circ}$, and the mean vertebral rotation angle at the apex was $37.7 \pm$ $20.8^{\circ}$. The mean spine CI of patients was $9.7 \pm 7.11$, which was significantly higher than that of the control group $(0.28 \pm 0.22, P=.00001)$.

Obtaining adequate quality $3 \mathrm{D}$ volume-rendered CT images for all patients was feasible in convenient time (4 to 10 minutes). Images show that the aorta becomes tortuous in scoliosis patients, following the curve of the spine either right or left, compared with the straight path of the aorta in normal control subjects (Figure 3).

The mean values and results of comparison between the aorta TI and aorta centerline angle of patients versus the control group are listed in Table 1 and graphically represented in Figure 4. The aorta was significantly more tortuous in scoliosis patients than in the control group, with significant differences observed between the 2 groups in aorta TI and aorta centerline angle.

In the patient group, the correlation between the aorta TI and Cobb's angle was excellent $(r=0.851$, $P=.00001)$. The derived linear regression equation was TI $=-4.92+0.18 \times$ Cobb's angle (Figure 5$)$. Similarly, there was excellent positive correlation between the aorta TI and each spine CI and vertebral rotation angle $(r=0.859, P=.0001 ; r=$ $0.867, P=.000007$, respectively). Excellent negative correlation was present between the aorta centerline angle and Cobb's angle $(r=-0.851, P=.000015)$, denoting a steeper centerline angle with increasing Cobb's angle. Among all, the spine CI and Cobb's angle had the highest correlation $(r=0.959, P=$ .000001).

The mean aorto-vertebral angles at each vertebral level for the control group and each of right and left scoliosis patients are listed in Table 2 (Figure 6). 


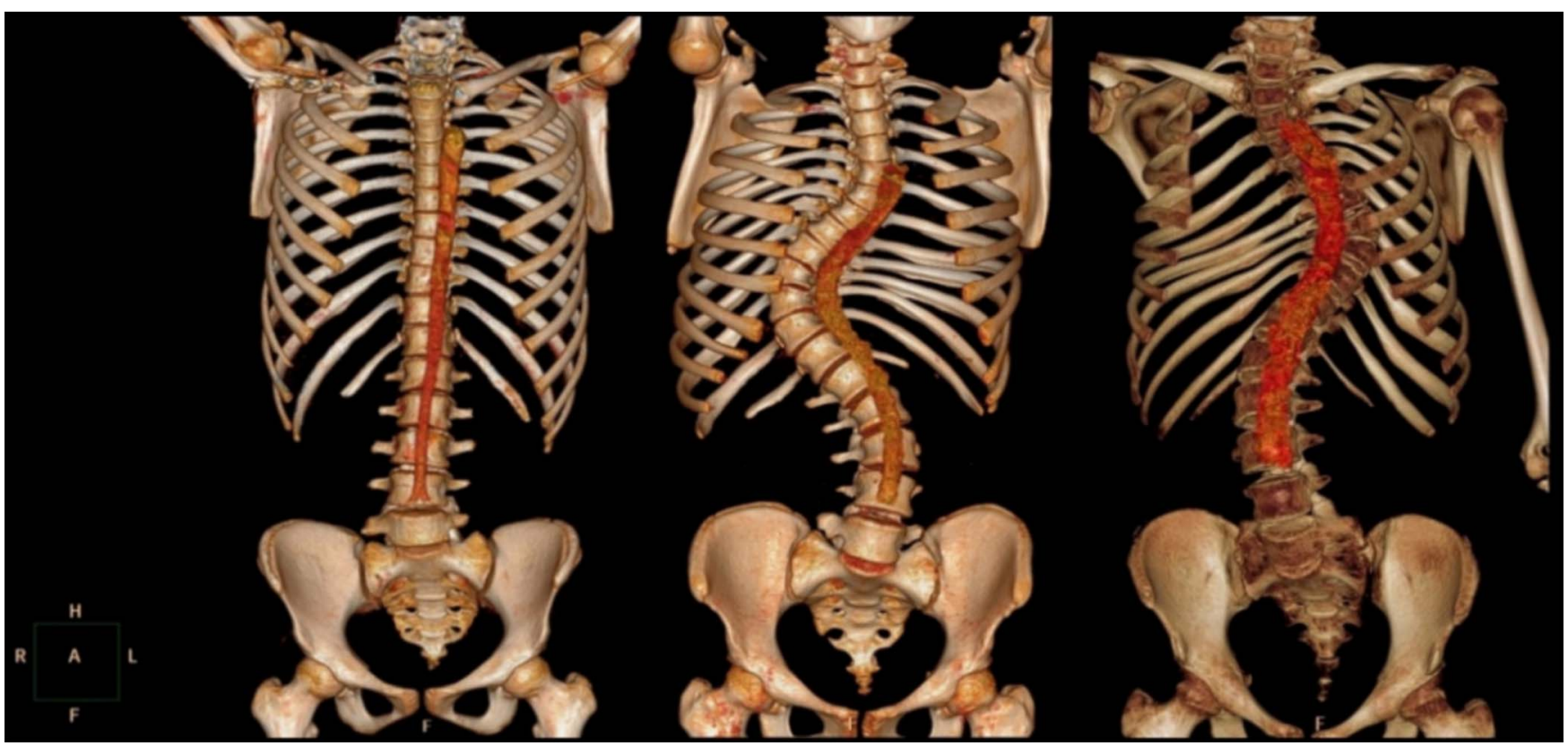

Figure 3. Examples of 3D volume-rendered reconstructions of the spine and the aorta in a control subject (right), scoliosis patient with right curve (middle), and scoliosis patient with left curve (left).

The aorto-vertebral angle was significantly different between the 3 groups at T6, T7, and T8 levels as well as at L2 and L3 levels.

\section{DISCUSSION}

The descending aorta is normally a straight conduit vessel, although some degree of aortic kinking or tortuosity is not uncommon, especially in the elderly population. ${ }^{16-18}$ Aortic kinking or tortuosity is linked to the multifactorial effect of aging, hypertension, diabetes, atherosclerosis, or genetic defects. ${ }^{19}$

Scoliosis represents another cause of aortic bending. In this condition, the aorta is presumably healthy, and the assumed mechanism of bending is much simpler. The descending aorta typically extends from opposite T4 down to its bifurcation opposite L4. In the presence of scoliosis, the vertical distance between T4 and L4 decreases; consequently, the aortic straight pathway becomes shorter than

Table 1. Aorta tortuosity index (TI) and aorta centerline angle in adolescent idiopathic scoliosis patients versus controls.

\begin{tabular}{lccc}
\hline & $\begin{array}{c}\text { Adolescent } \\
\text { Idiopathic Scoliosis } \\
\text { Patients, } \\
\text { mean } \pm \text { SD }(\boldsymbol{n}=\mathbf{1 7})\end{array}$ & $\begin{array}{c}\text { Control Group, } \\
\text { mean } \pm \text { SD } \\
(\boldsymbol{n}=\mathbf{1 1})\end{array}$ & $\boldsymbol{P}$ Value \\
\hline Aorta TI & $6.4 \pm 7.2$ & $0.6 \pm 0.5$ & $.0001^{\mathrm{b}}$ \\
Aorta centerline angle & $140 \pm 26.8$ & $170 \pm 3.6$ & $.0002^{\mathrm{b}}$ \\
\hline
\end{tabular}

Abbreviation: $\mathrm{SD}$, standard deviation.

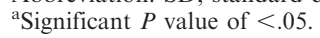

the actual length of the aorta, forcing it to assume a curved course.

In this study, we demonstrated both qualitatively and quantitatively the difference in aortic course and degree of aortic bending between scoliosis patients and controls. Qualitatively, the aorta followed the curve of the spine in scoliotic patients lying in the concavity of scoliosis, whether to the right or left. Quantitatively, the mean aorta TI of scoliosis patients was more than 10 times that of the control group. The mean aortic TI of 6.4 in scoliosis patients suggests that the centerline length (representing the actual aortic length) is $6.4 \%$ longer than the straight path between start and end points of the descending aorta. Another quantitative parameter, the centerline angle of the aorta, was significantly steeper in scoliosis patients than in the control group.

The degree of aortic bending in scoliosis patients, as measured by both TI and aorta centerline angle, had excellent correlation with the degree of spine curvature, as assessed by Cobb's angle. Few previous studies discussed the correlation between changes in the axial position of the aorta and severity of scoliosis. In one study, no correlation was found between Cobb's angle and aorto-vertebral angles, ${ }^{1}$ while in another study, the aorta position was significantly more lateral and posterior (higher aorto-vertebral angle) with higher Cobb's angle. $^{2}$ In a more recent study, a positive correlation 

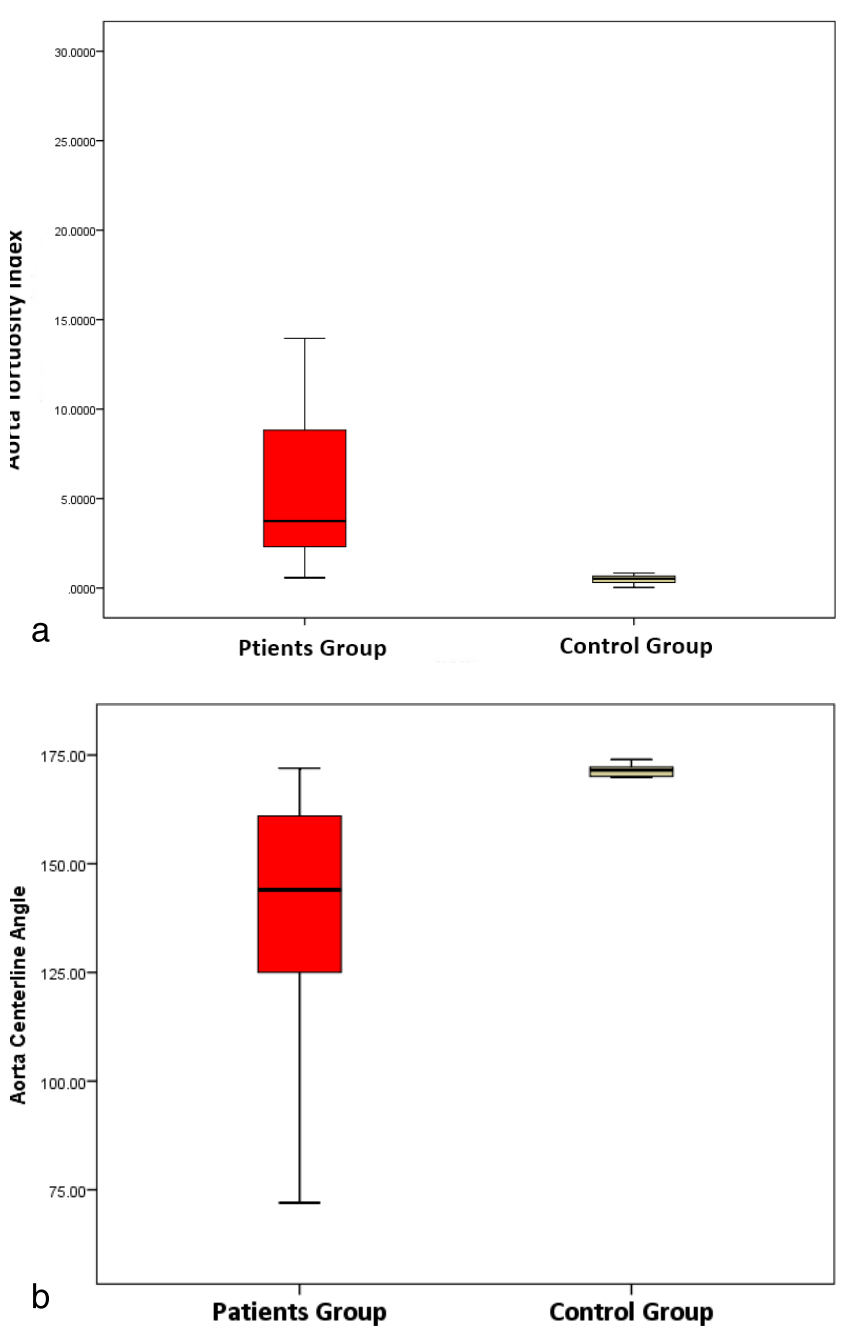

Figure 4. Box and whisker plots showing the distribution of aorta tortuosity index (a) and aorta centerline angle (b) in scoliosis patients compared with the control group.

was observed between aorto-vertebral distance and Cobb's angle, or, in other words, the larger the curve of scoliosis, the further the aorta from the apical spine. ${ }^{9}$

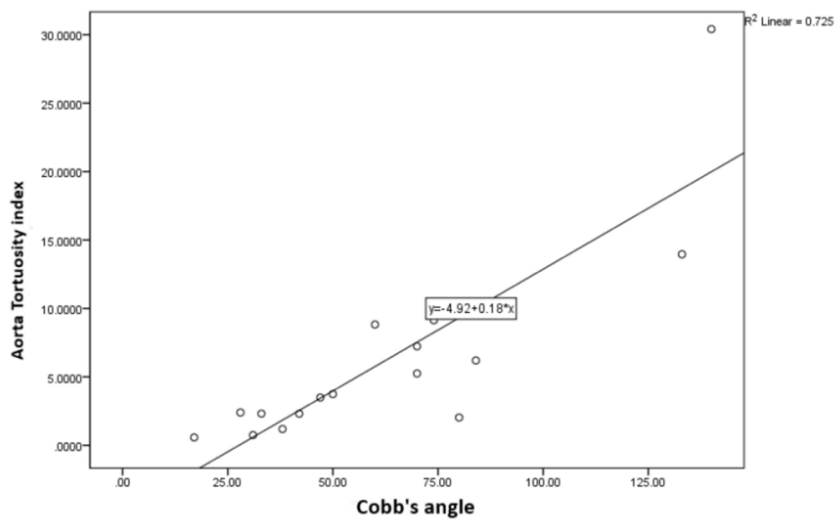

Figure 5. Scatter plot showing the distribution of aorta tortuosity index and Cobb's angle in the scoliosis patient group. The linear regression line and equation are shown.
Table 2. Mean aorto-vertebral (AV) angles at each vertebral level in patients and controls.

\begin{tabular}{|c|c|c|c|c|}
\hline $\begin{array}{l}\text { Vertebral } \\
\text { Level }\end{array}$ & $\begin{array}{c}\text { Adolescent } \\
\text { Idiopathic } \\
\text { Scoliosis Patients } \\
\text { (Right Curve) } \\
\text { AV Angle }\end{array}$ & $\begin{array}{c}\text { Adolescent } \\
\text { Idiopathic } \\
\text { Scoliosis Patients } \\
\text { (Left Curve) } \\
\text { AV Angle }\end{array}$ & $\begin{array}{c}\text { Control } \\
\text { Group } \\
\text { AV Angle }\end{array}$ & $P$ Value \\
\hline T4 & 47.9 & 37.8 & 48.4 & .810 \\
\hline T5 & 55.8 & 10.6 & 52.1 & .009 \\
\hline T6 & 69.9 & -8.5 & 51.1 & $.0001^{\mathrm{a}}$ \\
\hline $\mathrm{T} 7$ & 75.1 & -21 & 46.9 & $.000003^{\mathrm{a}}$ \\
\hline T8 & 62.4 & -15.8 & 41.5 & $.0003^{\mathrm{a}}$ \\
\hline T9 & 47.1 & -4.9 & 34.9 & .060 \\
\hline T10 & 42.7 & 13.6 & 30.1 & .262 \\
\hline T11 & 31.4 & 31.6 & 24.7 & .824 \\
\hline T12 & 19.9 & 34.1 & 24.8 & .713 \\
\hline L1 & 7.5 & 30.4 & 17.5 & .295 \\
\hline L2 & 1.6 & 29.7 & 13.2 & $.031^{\mathrm{a}}$ \\
\hline L3 & -4.8 & 22.7 & 10.9 & $.014^{\mathrm{a}}$ \\
\hline L4 & -1.7 & 13.4 & 12.6 & .416 \\
\hline
\end{tabular}

${ }^{a}$ Analysis of variance test, significant $P$ value of $<.05$.

We also calculated $\mathrm{CI}$ for the spine, using centerline and straight lengths in a similar fashion to the TI. One theoretical advantage of spine CI is that it takes into consideration the degree of each of the main and compensatory scoliotic curves together as well as any associated degree of kyphosis. The CI had excellent correlation to the Cobb's angle and to the aorta TI as well.

In our patients with right scoliosis curve (Figure 6), the axial aorto-vertebral angle increased gradually (the aorta was shifting in a postero-lateral direction to the left side) from $\mathrm{T} 4$ to reach the maximum angle at the $\mathrm{T} 7$, the most common apex level. Afterwards, the angle started to decrease gradually (the aorta was shifting in a right anteromedial direction) to end anteriorly just to the right of midline (small negative angle at L4). Several axial angles were used to describe the aorta position by different research groups, ${ }^{15}$ but despite this vari-

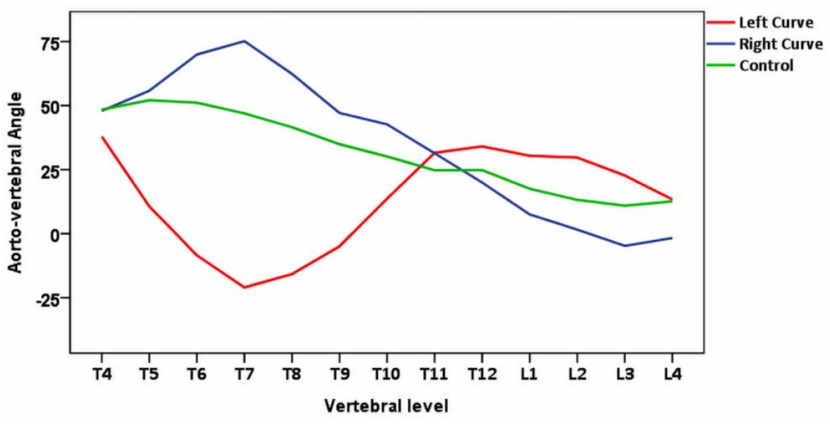

Figure 6. Line chart showing the mean aorto-vertebral angles measured at each vertebral level in the control group (green line), scoliosis patients with right curve (blue line), and scoliosis patients with left curve (red line). The 3 lines are furthest at the level of apex vertebra (T7) and closest at T11 to T12; the level of the diaphragmatic aortic hiatus is shown. 
ability in measurement methods, the net findings are similar.

Analyzing our patients with left scoliotic curve, the observed aorto-vertebral angle was less than the control at T4 and continued to decrease to a minimal negative value at $\mathrm{T} 7$ (the aorta was more anterior and to the right of the spine). The aortovertebral angle then started to increase gradually until the level of T11. These findings are comparable to the few studies that addressed the less common left scoliosis. 5,20

The tethering effect of the diaphragmatic hiatus on the aorta position mentioned in previous studies $^{5,20}$ was reflected in our aorto-vertebral angle measurements, where the least difference between right and left scoliosis patients and controls was observed at the D11/D12 level (the 3 lines are closest in Figure 6).

The main limitation of this study is the rather small sample size, especially in patients with left scoliotic curve. This prevented further subgrouping (eg, according to Lenke curve type). Postcorrection CT scans are not part of routine practice at our institution, so we could not investigate the migration of the aorta after surgical correction.

\section{CONCLUSION}

In conclusion, obtaining 3D volume-rendered images from preoperative noncontrast CT images of scoliosis patients may provide a useful overview of the whole course of the aorta and its relative position to the spine. In the axial plane, the aorta in both right and left scoliosis is maximally rotated away from its normal position at $\mathrm{T} 7$ (commonest apical level) and is closest to its normal position at T11 to T12 (diaphragmatic hiatus level). These reconstructed images could be beneficial for the surgeon in the planning of spine surgical procedures.

\section{REFERENCES}

1. Sevastik B, Xiong B, Hedlund R, Sevastik J. The position of the aorta in relation to the vertebra in patients with idiopathic thoracic scoliosis. Surg Radiol Anat. 1996;18(1):5156.

2. Sucato DJ, Duchene C. The position of the aorta relative to the spine: a comparison of patients with and without idiopathic scoliosis. J Bone Joint Surg Am. 2003;85(8):14611469.

3. Maruyama T, Takeshita K, Nakamura K, Kitagawa T. Spatial relations between the vertebral body and the thoracic aorta in adolescent idiopathic scoliosis. Spine (Phila Pa 1976). 2004;29(18):2067-2069.

4. Bullmann V, Fallenberg EM, Meier N, et al. The position of the aorta relative to the spine before and after anterior instrumentation in right thoracic scoliosis. Spine (Phila $\mathrm{Pa}$ 1976). 2006;31(15):1706-1713.

5. Milbrandt TA, Sucato DJ. The position of the aorta relative to the spine in patients with left thoracic scoliosis: a comparison with normal patients. Spine (Phila Pa 1976). 2007;32(12):348-353.

6. Takeshita K, Maruyama T, Ono T, et al. New parameters to represent the position of the aorta relative to the spine for pedicle screw placement. Eur Spine J. 2010;19(5):815-820.

7. Liu J, Shen J, Zhang J, et al. The position of the aorta relative to the spine for pedicle screw placement in the correction of idiopathic scoliosis. J Spinal Disord Tech. 2012;25(4):E103-E107.

8. Bekki H, Harimaya K, Matsumoto Y, et al. The position of the aorta relative to the vertebrae in patients with Lenke type 1 adolescent idiopathic scoliosis. Spine (Phila Pa 1976). 2016;41(7):585-590.

9. Chiu CK, Lee KJ, Chung WH, Chandren JR, Chan CYW, Kwan MK. Does the severity of the curve in Lenke 1 and 2 adolescent idiopathic scoliosis patients affect the distance and position of the aorta from vertebra? Spine (Phila Pa 1976). 2019;44(11):785-792.

10. Yang H, Liu Z, Guan L, Liu Y, Liu T, Hai Y. Is the risk of aorta injury or impingement higher during correction surgery in patients with severe and rigid scoliosis? World Neurosurg. 2020; 139: e626-634. doi:10.1016/j.wneu.2020.04.065

11. Lavigne F, Mascard E, Laurian C, Dubousset J, Wicart $P$. Delayed-iatrogenic injury of the thoracic aorta by an anterior spinal instrumentation. Eur Spine J. 2009;18(2):265-268.

12. Chan CYW, Kwan MK (2017) Safety of pedicle screws in adolescent idiopathic scoliosis surgery. Asian Spine J. 2009;11(6):998-1007.

13. Wang W, Zhu Z, Zhu F, et al. The changes of relative position of the thoracic aorta after anterior or posterior instrumentation of type I Lenke curve in adolescent idiopathic thoracic scoliosis. Eur Spine J. 2008;17(8):1019-1026.

14. Zhu F, Chen WJ, Wang WJ, et al. Migration of thoracic aorta after the anterior correction of thoracic idiopathic scoliosis without parietal pleura closure. J Spinal Disord Tech. 2011;24(6):390-396.

15. Yuan SM, Wang GR. Aortic issues in scoliosis and scoliotic operations. Wien Klin Wochenschr. 2016;128(3-4):131136.

16. Tawfik AM, Sobh DM, Gadelhak B, Sobh HM, Batouty NM. The effect of age and gender on tortuosity of the descending thoracic aorta. Eur J Radiol. 2019;110:54-59.

17. Adriaans BP, Heuts S, Gerretsen S, et al. Aortic elongation part $\mathrm{I}$ : the normal aortic ageing process. Heart. 2018;104(21):1772-1777.

18. Belvroy VM, de Beaufort HWL, van Herwaarden JA, Bismuth J, Moll FL, Trimarchi S. Tortuosity of the descending thoracic aorta: normal values by age. PLoS One. 2019;14(4): e0215549. doi: 10.1371/journal.pone.0215549. eCollection 2019.

19. Han HC. Twisted blood vessels: symptoms, etiology and biochemical mechanism. J Vasc Res. 2012;49(3):185-197.

20. Qiao J, Zhu F, Xu L, et al. Comparison of the aorta impingement risks between thoracolumbar/lumbar curves with different convexities in adolescent idiopathic scoliosis: a 
computed tomography study. Eur Spine J. 2012;21(10):20432049.

Declarations and COI: Not applicable.

Corresponding Author: Ahmed M. Tawfik, $\mathrm{MD}, \mathrm{PhD}$, Associate Professor of Radiology, Department of Diagnostic and Interventional Radiology, Mansoura University Hospital, 35112, 12 El-Gomhoreya Street, Mansoura, Egypt. Phone:
002-050-2222228; Email: ahm_m_tawfik@hotmail. com.

Published 19 August 2021

This manuscript is generously published free of charge by ISASS, the International Society for the Advancement of Spine Surgery. Copyright (C) 2021 ISASS. To see more or order reprints or permissions, see http://ijssurgery.com. 